Reprod. Nutr. Dévelop., 1985, 25 (4 A), 599-603.

\title{
Etude des enzymes plasmatiques des taureaux de combat tués en corridas (1)
}

\author{
A. PURROY UNANUA (2), J. M. GONZALEZ BUITRAGO $\left({ }^{*}\right)$
}

CRIDA 05, I.N.I.A., Apartado Oficial Salamanca, España

(*) Laboratorio de Bioquimica, Residencia Sanitaria "Virgen de la Vega ", Salamanca, España

Summary. A study of plasma enzymes in fighting bulls killed in Spanish bullfights.

Blood samples were taken from 50 fighting bulls used in several Spanish bullfights as soon as possible after the animals were killed. The blood was centrifuged and the plasma assayed for CK, GOT, LDH, GPT, $\gamma$-GT and for alkaline phosphatase activity.

The animals were grouped into three lots (A, B and $C$ ) according to the strength they showed during the bullfight. The results showed an inverse relationship between CK, GOT and LDH activities and bull strength during the bullfight, and significant differences were observed in CK activity among the three groups.

It was concluded that the high CK, GOT and LDH levels observed in groups B and C might be due to the fact either that the animals were subjected to more exercise and/or trauma during the days and hours preceding the bulfight or that some type of subclinical muscle pathology was present.

\section{Introduction.}

Depuis une vingtaine d'années environ, nous assistons lors des corridas à un spectacle pénible : des taureaux qui semblent être en très bonne condition tombent pendant le combat. Les aficionados et les professionnels de la tauromachie recherchent depuis longtemps les causes de ce phénomène.

La mesure de l'activité de diverses enzymes plasmatiques est un moyen de diagnostic ou de contrôle de l'évolution d'états pathologiques, utilisé chez I'homme (Moss et Butterworth, 1974 ; Wilkinson, 1976 ; Griffiths, 1979) ou chez l'animal (Boyd et al., 1964 ; Keller, 1971), notamment pour l'étude de la pathologie musculaire. Les enzymes les plus fréquemment dosées chez les bovins en cas de troubles pathologiques sont la glutamate oxaloacétate transaminase (GOT;

(1) Ce travail a été réalisé en marge du programme de recherche de I'Instituto Nacional de Investigaciones Agrarias (I.N.I.A., España).

(2) Adresse actuelle : CRIDA 03, I.N.I.A., Apartado 727, Zaragoza, España. 
E.C.2.6.1.1.), la glutamate pyruvate transaminase (GPT ; E.C.2.6.1.2), la lactate déshydrogénase (LDH; E.C.1.1.1.27) et ses isoenzymes, la créatine kinase (C.K. ; E.C.2.7.3.2.), la gammaglutamyl transpeptidase ( $\gamma$-GT ; E.C.2.3.2.1.) et la glutamate déshydrogénase (GLDH ; E.C.1.4.1.3.).

Ainsi, ont été dosés la GOT (Hidiroglou et al., 1967 ; Boyd et al., 1964), la LDH et ses isoenzymes (Keller, 1974a) et la CK (Keller et al., 1971; Dotta et Robutti, 1972 ; Anderson et al., 1976) chez les bovins. La CK est relativement spécifique du tissu musculaire (Keller, 1974b) et c'est pourquoi on a le plus souvent recours à son dosage dans le sérum ou le plasma lors de troubles musculaires, que ceux-ci soient d'origine génétique ou nutritionnelle.

Le présent travail a pour but de caractériser l'état physiologique des taureaux de combat et de comparer les résultats des analyses sanguines aux qualités présentées par les mêmes bêtes lors du combat précédant la prise d'échantillon. II a porté sur 50 taureaux mis à mort par estocade et les activités enzymatiques (CK, GOT, LDH, GPT, $\gamma$-GT et phosphatases alcalines) du plasma ont été rapprochées des notes obtenues au cours du combat (espagnol : lidia).

\section{Matériel et Méthodes}

L'étude porte sur 50 échantillons de sang prélevés et centrifugés dès que possible après la mise à mort des animaux.

Le sérum prélevé a été dilué à un taux correspondant aux concentrations requises pour les dosages. Ceux-ci ont été réalisés selon les méthodes optimisées, conformément aux recommandations de la German Society for Clinical Chemistry (1972). Les mesures ont été faites à $30^{\circ} \mathrm{C}$ et les résultats sont exprimés en unités/litre (u/l).

L'analyse statistique des résultats a été réalisée selon la méthode non paramétrique de Mann-Whitney pour des données non appariées.

La vigueur de chaque animal a été notée de manière subjective durant le combat et on a ainsi obtenu un classement en trois groupes :

$=$ animaux ayant une vigueur normale $(++)$,

$=$ animaux faibles $(+)$,

$=$ animaux très faibles $(-1$.

\section{Résultats}

Les 50 taureaux faisant l'objet de cette étude ont été toréés au cours de 8 corridas; dans chacune de celles-ci ne figuraient des bêtes que d'un seul et même élevage.

Ces taureaux ont été classés selon leur force physique et répartis en 3 groupes : $A$ : 19 animaux normaux $(++) ; B: 12$ animaux faibles $(+) ; C: 19$ animaux très faibles $(-)$.

La concentration en CK, GOT et LDH est d'autant plus élevée que les taureaux ont montré moins de vigueur au cours du combat (tabl. 1). 
TABLEAU 1

Activités moyennes des enzymes $C K$, GOT et $L D H$ des 3 groupes d'animaux (avec écart-type et niveaux de signification statistique)(1)

\begin{tabular}{|c|c|c|c|}
\hline & CK $(u / I)$ & GOT (u/l) & $\mathrm{LDH}(\mathrm{u} / \mathrm{l})$ \\
\hline Groupe $A(++)\langle n=19\}$ & $\begin{array}{c}1041 \pm 501 \\
P<0,01\end{array}$ & $\begin{array}{l}163 \pm 61 \\
P<0,05\end{array}$ & $\begin{array}{c}3013 \pm 627 \\
P<0,05\end{array}$ \\
\hline Groupe B $(+) \quad(n=12)$ & $\begin{array}{c}1916 \pm 568 \\
P<0,01\end{array}$ & $\begin{array}{c}203 \pm 80 \\
P<0,01\end{array}$ & $\begin{array}{c}3604 \pm 589 \\
P<0,01\end{array}$ \\
\hline Groupe C $(-) \quad(n=19)$ & $5152 \pm 3884$ & $327 \pm 134$ & $4838 \pm 2346$ \\
\hline
\end{tabular}

(1) Le niveau de signification entre 2 groupes est indiqué directement sur le tableau.

Les différences entre groupes sont toutes hautement significatives ( $P<0,01)$ pour $C K$. Pour GOT et $L D H$, les différences observées sont significatives $(P<0,05)$ entre les groupes $A$ et $B$, hautement significatives $(P<0,01)$ entre les groupes $B$ et $C$.

La répartition entre animaux normaux, faibles ou très faibles au cours des 8 corridas n'est pas aléatoire $\left(\chi^{2}=23,1 ; P<0,06\right)$, ce qui traduit des différences entre élevages d'origine.

Les teneurs moyennes en GPT, $\gamma$-GT et en phosphatases alcalines ne sont pas significativement différentes entre les 3 groupes de taureaux; c'est pourquoi nous avons rassemblé les données et présenté les valeurs moyennes dans le tableau 2.

\section{TABLEAU 2}

Activités moyennes de GPT, $\gamma$-GT et P. AL (phosphatases a/calines) de tous les animaux étudiés (avec écart-type).

\begin{tabular}{lr}
\hline & \multicolumn{1}{c}{$u / l$} \\
GPT $(n=50)$ & $41,8 \pm 19,4$ \\
$\gamma-\mathrm{GT} \quad(n=50)$ & $21,1 \pm 18,9$ \\
P.AL $(n=50)$ & $185,4 \pm 80,1$ \\
\hline
\end{tabular}

\section{Discussion}

Le tissu musculaire des bovins, comme celui des autres espèces, est particulièrement riche en créatine kinase (CK) (Keller, 1971, 1974b) et les états pathologiques dus à une altération des fibres musculaires se traduisent par des teneurs plasmatiques élevées en cette enzyme libérée par les cellules endommagées (Keller et al., 1971 ; Dotta et Robutti, 1972 ; Anderson et al., 1976).

Récemment, nous avons dosé CK dans le sang de bovins adultes et obtenu des concentrations comprises entre 200 et $300 \mathrm{u} / /$ (résultats non publiés), valeurs 
que l'on peut considérer comme normales compte tenu de la méthode de dosage et de la température de la mesure. Ces valeurs sont cependant légèrement supérieures à celles indiquées par Anderson et al. (1976) et par Baumgartner et al. (1980) pour l'espèce bovine.

La teneur moyenne en CK obtenue dans le groupe A lanimaux ayant eu un comportement normal), soit $1041 \pm 501 \mathrm{u} / \mathrm{l}$ est 4 fois plus élevée que la valeur de référence indiquée ci-dessus. Cette augmentation de teneur en CK peut être la conséquence des diverses manipulations subies par les animaux avant le combat (transport, mise en case, ...) et pendant celui-ci. Par contre, les valeurs observées sur les animaux des groupes B et C, respectivement 1916 et $5152 \mathrm{u} / \mathrm{l}$, ne sont pas dues seulement à l'effort au cours du combat et à la mise à mort, mais doivent avoir une autre origine. Trois animaux qui, du fait de leur grande faiblesse, furent renvoyés au corral sans combattre, et qui n'avaient donc pas subi d'agression physique, présentaient en effet des teneurs très élevées en CK : 3580,1460 et $16450 \mathrm{u} / 1$; ces 3 taureaux ont été abattus immédiatement après la corrida comme l'impose le règlement des spectacles taurins. Les teneurs en GOT et LDH, qui sont significativement différentes entre groupes et augmentent avec la faiblesse des animaux, confirment les résultats obtenus avec CK, montrant ainsi que les défauts présentés par certains animaux pendant la corrida proviendraient de troubles d'origine musculaire.

Les concentrations en GPT, $\gamma$-GT et phosphatases alcalines des sérums sont très voisines dans les 3 groupes et ne diffèrent pas de celles que nous avons déterminées chez d'autres bovins adultes (résultats non publiés).

Parmi les facteurs responsables des teneurs élevées en CK, GOT et LDH des animaux les plus faibles dans l'arène, nous pouvons retenir les deux suivants: - d'une part, dans les jours et les heures précédant la corrida, une plus ou moins grande agitation dont les causes sont absolument nouvelles pour l'animal, agitation associée ou non à des traumatismes d'importance variable ; - d'autre part, un état pathologique subclinique qui pourrait être dû à des carences en oligoéléments (en sélénium, par exemple). Cette carence, déjà envisagée par Roumengou (1973), entraînerait une dégénérescence musculaire (Lamand, 1978) se traduisant, dans le cas qui nous préoccupe, par des chutes au cours du combat.

Nos travaux en cours devraient permettre de déterminer l'éventuelle origine génétique ou nutritionnelle de ces chutes.

Reçu en mai 1983.

Accepté en mars 1985.

\section{Références}

ANDERSON Ph., BERRET S., PATTERSON D. S. P., 1976. The significance of elevated plasma creatine phosphokinase activity in muscle disease of cattle. J. Comp. Path., 86, 531-538.

BAUMGARTNER W., SCHLERKA G., PETSCHENIG W., 1980. Untersuchungen über die Blutgase, den Säure-Basen-Hauskalt, Elektrolytgekalt, einige Enzyme und Inkaltsstoff im Blut neugeborener Kälber. (II - Mitteilung : Elektrolytgekalte, Enzymaktivitäten und Gehalt an Gesamtbilirubin). Dtsch. tierärztr. Wschr., 87, 18-20. 
BOYD J. W., DOUGLAS T. A., GOULD G. H., GRINES F. C., 1964. The interpretation of serum enzyme assays in cattle. Vet. Rec., 76, 567-574.

DOTTA U., ROBUTTI B., 1972. A study on the serum levels of glutamic-oxalacetic and glutamicpyruvic transaminases, aldolase and creatine phosphokinase in normal calves and calves with muscular dystrophy. Folia veter. lat., 2, 310-325.

GRIFFITHS J. C., 1979. Clinical enzymology. Masson, New-York.

GERMAN SOCIETY FOR CLINICAL CHEMISTRY, 1972. Standardisation of methods for the estimation of enzyme activities in biological fluids. Z. Klin. Biochem., 10, 281-291.

HIDIROGLOU M., NELSON F. C., LESSARD J. R., TAYLOR P. A., 1967. Serum transaminase activity in calves affected by nutritional muscular dystrophy in Northern Ontario. Can. vet. J., 8, 62-69.

LAMAND M., 1978. Oligo-éléments, in Alimentation des ruminants. Ed. INRA Publications, Route de St Cyr 78000 Versailles.

KELLER P., 1971. Serumenzyme beim Rind: Organalysen und Normalwerte. Schweizer Archiv. Tierheilkunde, 113, 615-626.

KELLER P., 1974a. Lactate dehydrogenase isoenzymes in normal bovine serum and during experimental liver and muscle damage. Res. vet. Sci, 17, 49-58.

KELLER P., 1974b. Lactate dehydrogenase and its isoenzymes, creatine phosphokinase and aldolase in different bovine muscles. J. comp. Path., 84, 467-475.

KELLER P., GERBER H., MARTYNG J., 1971. Behaviour of serum enzymes in cattle with myopathy. Schweizer Archiv. Tierheilkunde, 113, 627-636.

MOSS D. W., BUTTERWORTH P. J., 1974. Enzymology and medicine. Pitman Medical, Londres.

ROUMENGOU M., 1973. Les chutes des taureaux de combat - Causes naturelles, causes frauduleuses, solutions. Chez l'auteur, 91300 Massy.

WILKINSON J. H., 1976. The principles and practice of diagnostic enzymology. Edward Arnold, Londres. 